\title{
O DESENVOLVIMENTO NEUROPSICOMOTOR EM CRIANÇAS COM CEGUEIRA CONGÊNITA: UM ESTUDO DE REVISÃO ${ }^{83}$ NEUROPSYCHOMOTIVE TIVE DEVELOPMENT IN CHILDREN WITH CONGENITAL BLINDNESS: A REVIEW STUDY
}

\author{
Isabella Carolina Barreto Blanco dos Santos ${ }^{84}$ \\ Ruth Maria Mariani Braz ${ }^{85}$
}

\begin{abstract}
RESUMO
Este artigo tem como objetivo divulgar o levantamento bibliográfico realizado sobre as peculiaridades do desenvolvimento neuropsicomotor em crianças com cegueira congênita. Para a realização deste levantamento optamos pelas bases científicas de dados Google Acadêmico, Bireme, Scielo, Lilacs. Os referenciais que encontramos mencionam que há defasagem entre o desenvolvimento neuropsicomotor em crianças com cegueira congênita e crianças com visão considerada normal. $O$ desenvolvimento neuropsicomotor é considerado como um processo sequencial contínuo e relacionado com a idade cronológica, pelo qual o ser humano adquire uma enorme quantidade de habilidades motoras. Entretanto são necessários mais trabalhos que abordem este tema.
\end{abstract}

Palavras chaves: Desenvolvimento Neuropsicomotor. Cegueira Congênita. Deficiência Visual. Inclusão.

\section{ABSTRACT}

This article aims to divulge the bibliographical study about the peculiarities of neuro motor development in children with congenital blindness. To carry out this survey we searched in the scientific bases of data Google Academic, Bireme, Scielo, Lilacs. The references we have found mention that there is a delay between neuro-motor development in children with congenital blindness and children with normal vision. Neuro motor development is considered as a continuous sequential process related to chronological age, by which the human being acquires an enormous amount of motor skills. However, more work is needed to address this issue.

Keywords: Neuro-motor development. Congenital blindness. Visual impairment. Inclusion.

\footnotetext{
${ }^{83}$ Parte da Dissertação de mestrado do CMPDI, projecto aprovado no comité de ética no do CAAE: 93552218.9 .0000 .5243$.

${ }^{84}$ Estudante do Curso de Mestrado Profissional em Diversidade e Inclusão - CMPDI- UFF; E-mail: isabellablancob@yahoo.com.br

85 Docente/pesquisador/colaborador do Curso de Mestrado Profissional em Diversidade e Inclusão CMPDI - UFF; E-mail:ruthmariani06@gmail.com
} 


\section{RevistAleph}

\section{INTRODUÇÃO}

No processo de desenvolvimento motor normal, a visão proporciona a integração das atividades motoras, perceptivas e mentais. As crianças com cegueira congênita apresentam, em sua grande maioria, um atraso considerável em seu desenvolvimento neuropsicomotor quando não estimuladas corretamente.

Da Silveira Nunes \& Lomonaco (2008) definem a cegueira congênita como:

A cegueira é uma deficiência visual caracterizada pela impossibilidade de apreensão de informações do mundo pela visão. Existem dois tipos de deficiência visual: cegueira e baixa visão. As duas formas mais comuns de avaliação da capacidade visual são pela acuidade (discriminação de formas) e pelo campo visual (capacidade de percepção da amplitude dos estímulos). Cego é o indivíduo com acuidade menor que 0,1 ou campo visual com menos de 20 graus. Já a baixa visão é definida por uma acuidade de 6/60 e 18/60 e/ou um campo visual entre 20 e 50 graus. A capacidade visual é avaliada por essas medidas com todas as correções ópticas possíveis (óculos, lentes etc.). A perda da visão antes dos cinco anos de idade é chamada cegueira congênita. Já os cegos que perdem a visão a partir dessa idade são considerados cegos adventícios (DA SILVEIRA, LOMONACO, 2008, p.1).

O desenvolvimento neuropsicomotor consiste em diversas áreas interdependentes, influenciadas por fatores biológicos como a idade gestacional e o peso da criança ao nascer, fatores socioambientais e herança genética (SANTOS et. al., 2009). É considerado como um processo sequencial contínuo e relacionado a idade cronológica, pelo qual o ser humano adquire uma enorme quantidade de habilidades motoras, que progridem de movimentos simples e desorganizados até à execução de habilidades motoras eminentemente organizadas (WILLRICH et al.; 2009).

A percepção visual é uma função bastante complexa, que ocorre em três fases: primária, secundária e terciária. Na primeira fase há captação da imagem pelos receptores fotossensíveis localizados na retina. Essa imagem é projetada no lobo occipital, onde se dá a recepção do estímulo visual. Na fase secundária ocorre o reconhecimento da imagem projetada e ela passa a ter significado, e na terciária ocorre uma integração cortical desta imagem com todos os outros sentidos. Portanto, a visão está estreitamente correlacionada com outras atividades sensoriais (FIGUEIRA, 2000). 


\section{RevistAleph}

Segundo Couto Júnior \& Oliveira (2016) a principal causa para cegueira é a retinopatia da prematuridade, seguida pela atrofia do nervo óptico e em terceiro lugar pelo glaucoma congênito. A criança com cegueira congênita apresenta a deficiência no momento do nascimento ou em período imediato. Acredita-se que existem no mundo 1,5 milhões de crianças cegas e destas $75 \%$ são devidos a causas preveníveis como a retinopatia da prematuridade (GONÇALVES et. al., 2014).

A integridade do desenvolvimento neuropsicomotor depende da organização dos sentidos pelo sistema nervoso central, sendo este a base para potencializar o desenvolvimento das habilidades humanas, do comportamento e da aprendizagem. $\mathrm{O}$ desenvolvimento neuropsicomotor acontece pela satisfação da criança ao experimentar algo novo como uma aquisição motora ou sensorial. No processo de desenvolvimento motor normal, a visão proporciona a integração das atividades motoras, perceptivas e mentais. Aproximadamente 80 \% das informações são recebidas por este sentido, por isso a cegueira não é apenas a ausência de um sentido isolado, mas do sentido que integra todos os outros, o que afeta a criança no seu desenvolvimento neuropsicomotor (SOUZA et.al; 2010; FIGUEIRA, 2000).

Esta pesquisa justifica-se devido ao aumento do número de neonatos com sequelas de toda ordem, inclusive sensoriais, decorrente do avanço da medicina atual e da escassez de pesquisas atualizadas relacionadas com 0 desenvolvimento neuropsicomotor de crianças com cegueira congênita. Com isso, essa pesquisa pretende divulgar através da revisão de literatura as peculiaridades do desenvolvimento neuropsicomotor em crianças com cegueira congênita.

\section{METODOLOGIA}

O presente trabalho é uma revisão de literatura de caráter descritivo, qualitativo, com o objetivo de evidenciar as peculiaridades do desenvolvimento neuropsicomotor da criança com cegueira congênita, para que assim possam ser estimuladas adequadamente, minimizando as desigualdades quando comparadas com crianças com a visão normal. Sendo assim, iniciamos a busca por produções científicas 


\section{RevistAleph}

nas bases de dados Google Acadêmico, Bireme e Scielo, referentes a desenvolvimento neuropsicomotor, cegueira congênita, deficiência visual e criança, a fim de perceber se há trabalhos e pesquisas voltadas para essa temática. Os textos foram analisados, tendo suas principais informações sintetizadas com o objetivo de obter informações consistentes.

\section{RESULTADOS E DISCUSSÃO}

Incialmente no levantamento bibliográfico que realizamos nas bases de dados com o descritor "desenvolvimento neuropsicomotor", a pesquisa encontrou 2.800 trabalhos no Scielo, 10.500 no Google Acadêmico e 436 no Bireme. Entretanto quando acrescentamos os descritores "cegueira congênita, deficiência visual e criança", junto com desenvolvimento neuropsicomotor, reduzimos para 233 artigos no Scielo, 3.840 no Google Acadêmico e 3 no Bireme relacionados a nossa área de pesquisa, conforme o gráfico 1.

Gráfico 1- Números de artigos encontrados nas bases de dados.

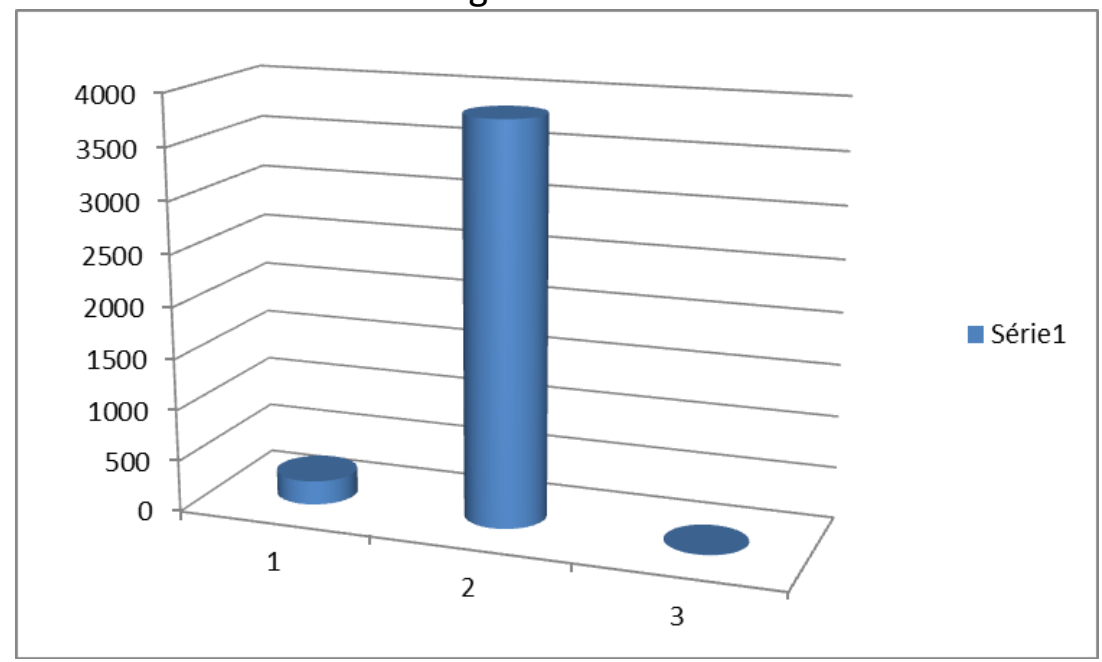

Fonte: Arquivo pessoal

Após análise dos títulos e dos resumos apresentados, dentre os trabalhos encontrados, apenas 20 estavam dentro do objetivo do presente estudo. Desta maneira, percebemos que não há muitas produções recentes que envolvam esta temática. 


\section{RevistAleph}

Estudos como os de Costa e Padilha, (2011), Pinheiro, et.al. (2010), Miranda et.al., (2010), Neto \& Rodrigues (2010) mostraram o aumento de sobrevivência de crianças com deficiência decorrente do avanço tecnológico da Medicina atual. Com isso o número de crianças com deficiência visual associada à deficiência física, auditiva e intelectual inseridas na educação também vem aumentando consideravelmente. Logo, a proposta da inclusão escolar desses alunos tem sido um tema em discussão em diferentes trabalhos pesquisados, no intuito de se compreender e viabilizar uma educação efetiva e de qualidade para todos os alunos, de acordo com suas características individuais. Entretanto, ainda há necessidade de ações voltadas para o cumprimento do direito à educação, especificamente na inclusão escolar de alunos com deficiências, devido ao despreparo de profissionais e à escassez de serviços e recursos de apoio que favoreçam a qualidade de ensino (MEDEIROS E BECKER, 2009).

Portanto, percebemos a necessidade de seguir a indagação por produções científicas dentro dos descritores apresentados e selecionando outros, assim como outras bases de dados, e percebendo a importância de trabalhos que possam envolver o desenvolvimento neuropsicomotor e a cegueira congênita primordialmente.

As novas teorias do desenvolvimento motor não se fundamentam apenas no sistema nervoso central como a causa. Outros sistemas como o sistema musculoesquelético, cardiorrespiratório e o ambiente também são responsáveis por exercer funções nesse processo. A teoria dos sistemas reconhece causas múltiplas e complexas do desenvolvimento. Embora um fator possa ser identificado como um catalisador para mudanças, nenhum agente sozinho pode ser considerado a origem do desenvolvimento motor. Todos os sistemas se submetem constantemente a mudanças, sendo assim dinâmicos. É a interação desses sistemas dinâmicos que promove o desenvolvimento das habilidades motoras (TECKLIN, 2010, p. 33).

Com isso, o desenvolvimento neuropsicomotor está diretamente ligado à interação dos fatores genéticos e biológicos com o meio que o cerca, e se por algum motivo essas interações não ocorrerem de forma eficiente, acontecerá um déficit no desenvolvimento dessa criança (SILVA et. al.; 2017). 


\section{RevistAleph}

Desde as primeiras horas de vida buscamos estímulos e respostas do ambiente, todos os sistemas trabalham para nos adaptar aos fatores ambientais. Distúrbios sensoriais podem afetar o desenvolvimento motor da criança. Os sentidos são de suma importância nas relações que a criança mantém com o mundo, principalmente no início do seu desenvolvimento, formando assim a base para todas as suas futuras aquisições (PADULA \& SPUNGIN, 2000; RODRIGUES, 2002).

Neste contexto, a visão desempenha um papel fundamental no sistema sensorial e é um dos principais canais para o desenvolvimento neuropsicomotor da criança. Favorece sua interação com o meio e estimula a comunicação, locomoção, mobilidade, orientação, controle de ações e percepções, além de proporcionar informações constantes e verificação imediata (FAVILLA et. al., 2014; SANTIN \& SIMMONS, 2000).

A visão está diretamente relacionada ao desenvolvimento, com isso a redução das capacidades visuais pode comprometer a funcionalidade e a independência da criança, resultando em comprometimentos em diversas áreas do desenvolvimento infantil. Grande parte das experiências da criança na exploração do ambiente, na descoberta do próprio corpo, na relação com os objetos e com outras pessoas, tem participação direta da visão, que se torna um elemento fundamental na efetivação das ações da criança em seu meio ambiente (MANCINI et. al.; 2010).

Por isso, crianças com cegueira congênita apresentam, em sua grande maioria, um atraso considerável em seu desenvolvimento neuropsicomotor quando não estimuladas corretamente. São fatores principais para o desenvolvimento o grau da deficiência e a idade em que ocorreu, e principalmente o tipo de tratamento e intervenção, uma vez que sabemos que quanto mais cedo o déficit for diagnosticado e tratado, melhores são as possibilidades de minimizar o atraso do desenvolvimento, deixando a criança o mais funcional possível (HOFFMANN, 1997; DIAS \& GALLO, 2010).

Os fatores socioeconômicos como grau de escolaridade e renda familiar, tempo disponível para a convivência familiar e materna, saneamento básico, noções de higiene pessoal e alimentar, atenção preventiva de manutenção da saúde ou de tratamento precoce de doenças, são considerados fatores de influência relacionados com a oferta 


\section{RevistAleph}

de experiências a essas crianças e também devem ser levados em consideração (BERNARDI \& COSTA, 2018).

Considerando que a visão fornece quase que instantaneamente a informação sobre ambientes próximos e distantes, e ainda é utilizada para regular a locomoção e o planejamento do deslocamento, garantindo assim a estabilidade corporal, percebemos sua importância para aprendizagem e desenvolvimento motor, dificultado quando esta não está disponível, principalmente na infância (MEERREIS et. al.; 2011).

A cegueira, quando presente no nascimento, afeta o desenvolvimento da percepção espacial e de distância, da identificação das qualidades dos objetos, do desenvolvimento das habilidades motoras e do comportamento social. Os estímulos auditivos e táteis são vistos como meios de exploração e interação para as crianças com deficiência visual. É através desses estímulos que essas crianças formam suas imagens, que possibilitam o conhecimento do mundo que as cerca (FIGUEIRA, 2002; KREUTZ \& BOSA, 2009).

Entretanto, o processamento de informações que essas crianças recebem se torna limitado não só pela quantidade de informações recebida do meio, mas principalmente pela qualidade com que essas informações são recebidas. O mundo visual é mais atraente, nem todos os objetos apresentam odor ou som, mas todos têm cores e formas. Assim, essas crianças acabam sendo prejudicadas em relação às crianças com visão considerada normal (OLIVEIRA FILHO et. al.; 2006).

Ainda assim, a criança com cegueira congênita tem uma capacidade contínua de se adaptar à sua deficiência se estimulada corretamente. A deficiência visual não pode ser considerada como causa de déficits cognitivos, motores ou psíquicos, mas sim como um fator predisponente à instalação desses quadros (FIGUEIRA, 2000).

O desenvolvimento neuropsicomotor em crianças com cegueira congênita, quando comparado ao de crianças que enxergam, é o mesmo, mas com um ritmo mais lento para a aquisição de postura e deslocamento, além da dificuldade na construção do esquema corporal (BERNARDI \& COSTA, 2008).

Entretanto, o sentimento de piedade é frequente em relação à criança com cegueira congênita fazendo com que a família a proteja excessivamente em casa, 


\title{
RevistAleph
}

evitando que brinque com outras crianças ou que ande e descubra o ambiente para que não se machuque, o que interfere diretamente na independência dessas crianças para desempenhar suas atividades. Por isso, durante a estimulação precoce se faz necessário que os cuidadores sejam orientados a dar o máximo de independência e liberdade possíveis a essas crianças (MALTA et. al.; 2006).

Dessa forma a identificação e a intervenção precoce são essenciais para o melhor desempenho das crianças com cegueira congênita, visto que as principais modificações do comportamento visual ocorrem nos primeiros meses de vida. Durante esse período há grande influência da neuroplasticidade que é induzida pela interação entre fatores genéticos e experiências ambientais (FAVILLA et. al.; 2014).

\begin{abstract}
A intervenção precoce é um processo que se estabelece com a parceria entre médicos, terapeutas e a família. A relação de confiança estabelecida desde os primeiros contatos firma a base para a habilitação da criança. Intervir precocemente requer profundo conhecimento sobre o processo de desenvolvimento humano, mais especificamente sobre o processo de desenvolvimento da criança e dos fatores que nele interferem. Além disso, a habilitação de crianças deficientes visuais requer compreensão das limitações visuais e, sobretudo, das capacidades visuais dessas crianças e possibilidades de desenvolvimento da eficiência da visão (GALIARDO \& NOBRE, 2001 p.16-17).
\end{abstract}

A Intervenção Precoce tem como princípios a estimulação e a integração dos sentidos remanescentes como a audição e o tato, para que assim o desenvolvimento neuropsicomotor dessas crianças seja o mais próximo possível do normal (RODRIGUES \& NACÁRIO, 2006).

Neste contexto observamos que a partir do conhecimento das peculiaridades do desenvolvimento neuropsicomotor em crianças com cegueira congênita, conseguimos atuar com intervenções que vão minimizar esta defasagem em relação às crianças com o desenvolvimento considerado normal. Esta estimulação pode ser realizada em diferentes espaços como nas escolas, especificamente nas salas de recursos multifuncionais, como também em clínicas ou com profissionais especializados. 


\section{RevistAleph}

\section{CONSIDERAÇÕES FINAIS}

Concluímos com esta revisão que a visão proporciona a integração das atividades motoras, perceptivas e mentais. Neste contexto a visão é um dos principais canais para o desenvolvimento neuropsicomotor. Com isso, quando a cegueira está presente desde o nascimento, percebemos um déficit quanto ao desenvolvimento nas habilidades neuropsicomotoras. Para estas crianças os estímulos auditivos e táteis são vistos como meios de exploração e interação, pois é através desses estímulos que elas conhecem o mundo que as cerca. Contudo, embora exista um atraso significativo em relação ao desenvolvimento neuropsicomotor de crianças com cegueira congênita quando comparado com o de crianças com visão considerada normal, após identificação e intervenção precoce, essa defasagem pode ser minimizada. Desta maneira, autores como Malta et.al., (2006) e Favilla et.al., (2014) apontam que após intervenções específicas é possível reduzir o atraso do desenvolvimento neuropsicomotor dessas crianças quando comparadas ao de crianças com visão normal. Entretanto, ainda se fazem necessárias mais pesquisas que correlacionem os descritores abordados nesta pesquisa.

\section{REFERÊNCIAS}

ALMEIDA, TAMIRES. S.; ARAÚJO, FILIPE. V. Diferenças experienciais entre pessoas com cegueira congênita e adquirida: uma breve apreciação. Revista Interfaces: Saúde, Humanas e Tecnologia. Ano 1, v. 1, n.3, jun, 2013.

BERNARDI, DANIELA F.; COSTA JULIANA. A criança cega: uma ação multidisciplinar com enfoque escolar. Anuário da Produção Acadêmica Docente, Vol. II, №. 3, Ano 2008, p. 133-145.

COSTA R. \& PADILHA M. I. A unidade de terapia intensiva neonatal possibilitando novas práticas no cuidado do recém-nascido. Revista Gaúcha de Enfermagem, Porto Alegre (RS) 2011, jun; 32(2): 248-55.

COUTO JUNIOR ABELARDO, OLIVEIRA LUCAS A. G. As principais causas de cegueira e baixa visão em escola para deficientes visuais. Rev Bras Oftalmol.2016; 75 (1): 26-9. 


\section{RevistAleph}

DA SILVEIRA NUNES, Sylvia; LOMÔNACO, José Fernando Bitencourt. Desenvolvimento de conceitos em cegos congênitos: caminhos de aquisição do conhecimento. Psicologia escolar e educacional, 2008, 12.1: 119-138.

FAVILLA MARCELO, CRUZ ANA FLAVIA I., MARTINS PAULA, ARRUDA SONIA M.C.P., GAGLIARDO HELOISA G.R.G. Avaliação da visão funcional de uma criança prematura com cegueira congênita. Cad. Ter. Ocup. UFSCar, São Carlos, v. 22, n. 2, p. 429-434, 2014.

GAGLIARDO, Heloisa GR Gardon; NOBRE, Maria Inês RS. Intervenção precoce na criança com baixa visão. Revista Neurociências, 2001, 9.1: 16-19.

GONÇALVES MARIA C.P.; SOARES TATIANA C.; SANTANA LUZICACIA M. Efeitos da fisioterapia no desenvolvimento motor do lactente com deficiência visual total. Revista Eletrônica Estácio Saúde - Volume 3, Número 1, 2014.

GRAZIANO ROSA. M; LEONE CLEA.R. Problemas oftalmológicos mais frequentes e desenvolvimento visual do pré-termo extremo. Jornal de Pediatria - Vol. 81, №1(supl), 2005.

HOFFMANN SONIA B. Dificuldades no desenvolvimento motor e a orientação e mobilidade da criança cega. Perfil - Ano 01- Na1 - 1997.

KREUTZ CARLA M.; BOSA CLEONICE A. Intervenção precoce na comunicação pais- bebê com deficiência visual. Estudos de psicologia, Campinas 26(4) 537-544 outubrodezembro 2009.

MALTA JULIANA; ENDRISS DANIELA; RACHEAD SUELI; MOURA TEREZINHA; VENTURA LIANA. Desempenho funcional de crianças com deficiência visual, atendidas no Departamento de Estimulação Visual da Fundação Altino Ventura. Arq Bras Oftalmol. 2006;69(4):571-4.

MANCINI, M. C.; BRAGA, M. A. F.; ALBUQUERQUE, K. A.; RAMOS, T. M. V.; CHAGAS, P. S. C. Comparação do desempenho funcional de crianças com visão subnormal e crianças com desenvolvimento normal aos 2 e 6 anos de idade. Rev. Ter. Ocup. Univ. São Paulo, v. 21, n. 3, p. 215-222, set./dez. 2010.

MEEREIS ECW, LEMOS LFC, PRANKE GI, ALVES RF,TEIXEIRA CS, MOTA CB. Deficiência visual: uma revisão focada no equilíbrio postural, desenvolvimento psicomotor e intervenções. R. bras. Ci. e Mov 2011;19(1):108-113.

MIRANDA A. M., CUNHA D. I. , GOMES S. M. F. A influência da tecnologia na sobrevida do recém-nascido prematuro extremo de muito baixo peso. Rev. Min. Enferm.; 14(3): 435-442, jul./set., 2010.

NETO J. A. S. \& RODRIGUES B. M. R. D. Tecnologia como fundamento do cuidar em neonatologia. Texto Contexto Enfermagem. Florianópolis, 2010 abr-jun; 19(2): 372-7 


\section{RevistAleph}

OLIVEIRA FILHO CIRO.W.; MATARUNA LEONARDO; CARVALHO ARTHUR J.S.; ARAÚJO PAULO F.; ALMEIDA JOSÉ J.G.; As relações do jogo e o desenvolvimento motor na pessoa com deficiência visual. Rev. Bras. Cienc. Esporte, Campinas, v. 27, n. 2, p. 131147, jan. 2006.

PINHEIRO C. E. A., PERES M. A., ORSI E. Aumento da sobrevida de crianças de grupos de peso baixo ao nascer em Santa Catarina. Revista Saúde Pública 2010, 44(5): 77684.

RODRIGUES MARIA RITA C. Estimulação precoce: A contribuição da psicomotricidade na intervenção fisioterápica como prevenção de atrasos motores na criança cega congênita nos dois primeiros anos de vida. Revista Benjamin Constant. no04, p. 3-5. Rio de Janeiro: Ministério da Educação e do Desporto, 2006.

SANTOS D.C.C.; TOLOCKA R.E; CARVALHO J.; HERINGER L.R.C.; ALMEIDA C.M.; MIQUELOTE A.F. Desempenho motor e fatores neonatais, familiares e exposição a creche. Rev Bras Fisioter. 2009;13(2):173-9.

SILVA RAFAELA E. G.; PEREIRA CRISTIANE; SILVA DAIANA F.; CARVALHO REBECCA O. R. Avaliação do desenvolvimento neuropsicomotor em crianças de 4 meses a 3 anos de duas creches da cidade de Porto Velho - RO. SOUTH AMERICAN Journal of Basic Education, Technical and Technological. ISSN:2446-4821. Vol. 4 N. 1 (2017) P. 106117.

SILVA TATIANA M.; VARGAS PATRÍCIA L. O lúdico e a aprendizagem da pessoa com deficiência visual. REVISTA PÓS-GRADUAÇÃO: DESAFIOS CONTEMPORÂNEOS v.1, n. 1 , jun/2014

SOARES ANTONIO V. A contribuição visual para o controle postural. Rev Neurocienc 2010;18(3):370-379.

SOUZA TELMA A.; SOUZA VIVIAN E.; LOPES MARCIA. C. B.; KITADA SILVIA P.S. Descrição do desenvolvimento neuropsicomotor e visual de crianças com deficiência visual. Arq Bras Oftalmol . 2010;73(6):526-30.

TECKLIN J. S.; Fisioterapia Pediátrica. Ed. ARTMED. 2010, 480p.

WILLRICH ALINE; AZEVEDO CAMILA C. F.; FERNANDES JULIANA O. Desenvolvimento motor na infância: influência dos fatores de risco e programas de intervenção. Re.

Neurocienc 2008: 17(1):51

Data de envio:21/05/2019

Data de aceite:13/12/2019 